This item was submitted to Loughborough's Research Repository by the author.

Items in Figshare are protected by copyright, with all rights reserved, unless otherwise indicated.

\title{
Examining lane change gap acceptance, duration and impact using naturalistic driving data
}

PLEASE CITE THE PUBLISHED VERSION

https://doi.org/10.1016/j.trc.2019.05.024

PUBLISHER

(c) Elsevier

VERSION

AM (Accepted Manuscript)

\section{PUBLISHER STATEMENT}

This paper was accepted for publication in the journal Transportation Research Part C: Emerging Technologies and the definitive published version is available at https://doi.org/10.1016/j.trc.2019.05.024.

\section{LICENCE}

CC BY-NC-ND 4.0

\section{REPOSITORY RECORD}

Yang, Minming, Xuesong Wang, and Mohammed Quddus. 2019. "Examining Lane Change Gap Acceptance, Duration and Impact Using Naturalistic Driving Data". Loughborough University.

https://hdl.handle.net/2134/37855. 


\title{
Examining Lane Change Gap Acceptance, Duration and Impact
}

\section{Using Naturalistic Driving Data}

\author{
Minming Yang ${ }^{\mathrm{a}, \mathrm{b}}$, Xuesong Wang ${ }^{\mathrm{a}, \mathrm{b}^{*}}$, Mohammed Quddus ${ }^{\mathrm{c}}$ \\ ${ }^{a}$ The Key Laboratory of Road and Traffic Engineering, Ministry of Education, Shanghai, 201804, China \\ ${ }^{b}$ School of Transportation Engineering, Tongji University, Shanghai, 201804, China \\ ${ }^{c}$ School of Civil \& Building Engineering, Loughborough University, Loughborough LE11 3TU, United \\ Kingdom \\ *Corresponding Author.Tel: +86-21-69583946.E-mail address: wangxs@tongji.edu.cn
}

\begin{abstract}
:
Analysis of lane change is important for microsimulation and safety improvement, and can also provide reference for advanced driver assistance systems (ADAS) and connected and autonomous vehicles (CAVs). Yet little research has comprehensively explored lane changing, particularly in China, a site of current CAV testing. This study developed an automatic extraction algorithm to retrieve 5,339 lane change events from the Shanghai Naturalistic Driving Study, and used the data to examine the core lane change components: gap acceptance, duration, and impact on the following vehicle (FV). Multilevel mixed-effects linear models were employed to develop relationships between gap acceptance and duration and the influencing factors; impact was then assessed using speed change rate, brake timestamping, and time-to-collision (TTC). Key results showed that 1) gap acceptance varied by roadway type and motivation, and lead and lag gaps were significantly affected by environmental variables, vehicle type, and kinematic parameters; 2) duration varied from $0.7 \mathrm{~s}$ to $16.1 \mathrm{~s}$, significantly affected by variables similar to gap acceptance, but notably, not by motivation; 3 ) as many as 1 in 5 Chinese FV drivers responded to lane changes with acceleration exceeding $10 \%$; 4) nearly half of FVs braked when they perceived a vehicle's lane-change intention, and $90 \%$ braked before TTC reached $4.7 \mathrm{~s} ; 5$ ) in over $70 \%$ of lane changes, the minimum TTC occurred between the initiation and cross-lane points. In addition to advancing the international development of lane-change theory, one of this study's important applications is that CAVs can be designed to brake during a safer TTC phase.
\end{abstract}

Keywords: Lane change behavior; Naturalistic driving study; Gap acceptance; Duration model; Lane change impact; ADAS and CAVs 


\section{Introduction}

Roadway lane changes are common maneuvers, but they have substantial impact on traffic flow. Previous studies have shown that lane changing tends to cause negative shockwaves (Cassidy and Bertini, 1999), and particularly risky lane changes may lead to severe traffic collisions. Unsafe and improper lane changes have been reported to account for $4.9 \%$ of all traffic crashes in China in 2015 (Traffic Management Bureau of the Public Security Ministry, 2016). Analyzing lane change behavior is therefore important for several applications, such as roadway capacity modeling and safety studies (Toledo et al., 2003), but also has other benefits. Since the emergence of traffic and driving simulation tools for transportation system analysis, interest in lane change behavior has increased, as realistic representations of complex realworld phenomena is fundamental to traffic microsimulation (Park et al, 2015). Lane change also plays an important role in the development of advanced driver assistance systems (ADAS) and connected and autonomous vehicles (CAVs) (Zhou and Peng, 2015; Cao et al, 2017). With accurate and robust lane change decision-making, planning, and controlling systems, ADAS and CAVs can make progress toward achieving global optimization objectives such as improving traffic safety, promoting fuel economy, easing urban traffic congestion and optimizing the use of roadways (Man, 2007).

A driver goes through several stages during a lane change: decision-making, preparation, execution and interaction with surrounding vehicles (Nie et al, 2017). Existing lane-change studies roughly fall into two categories of analysis: (i) lane change decision-making (i.e., how a driver reaches the lane change decision when facing a specific scenario) and execution processes (including duration), and (ii) lane change impact on surrounding vehicles (e.g., how drivers of following vehicles respond to lane changes) (Zheng, 2014). Most studies, however, focus only on either the decision-making and execution processes or on lane change impact, so a comprehensive understanding of the full lane change process and its implications remains, for the most part, elusive. As both of these categories, each of which influences the other, are critical for theoretical modeling and application, this study aims to provide an overall perspective on lane change behavior by examining its three core components: gap acceptance, duration and impact on the following vehicle (Zheng, 2014).

Another objective of this study is to offer insight into lane change behavior, specifically in China. While it is well known that China's traffic systems have developed rapidly during recent years, few studies of driving behavior have analyzed lane change maneuvers in China due to limited data collection methods. Therefore, most simulation models are based on research from developed nations, where the cultural environment, including driving style, vehicle type, and traffic regulations, may differ from developing countries (Lindgren et al, 2008). Chinese drivers face, for example, a challenging driving environment of omnipresent pedestrians, electric bikes, bicycles, aggressive drivers, and, indeed, frequent lane changes, which are performed nearly three times more often than in the U.S. (Wang and Li, 2016). In addition, some ADAS and CAV functions may not be suitable in China, as their algorithms and strategies are calibrated and tested using data from other developed nations. This is not only a problem for Chinese traffic safety. The increased CAV testing in China makes accounting for these differences essential to more effective and robust advanced traffic technologies.

To address these needs, real-world driving data were collected in the Naturalistic Driving Study conducted in Shanghai (SH-NDS) from December 2012 to December 2015. During the study period, 60 licensed drivers travelled approximately 161,055 km (Wang et al, 2019; Zhu 
1 et al, 2018). Using the NDS's significant quantity of Chinese driving data, this study comprehensively explores lane change behavior to contribute to the international development of lane change theory and its various applications, including traffic simulation, ADAS, and CAV.

\section{Literature review}

\subsection{Gap acceptance}

Gipps (1986) considered necessity, desirability and safety in the first lane change model in microsimulation software; but in all models, gap acceptance is a crucial element in the decisionmaking process. Drivers considering a lane change decide whether to accept an available gap, i.e., they assess whether the longitudinal gaps between their own vehicle and the vehicles in the target lane are sufficient. The target gap is separated into the lead gap, the longitudinal distance between the lead vehicle (LV) in the target lane and lane changing vehicle (LCV); and the lag gap, the distance between the following vehicle (FV) and the LCV (Toledo et al., 2003). In this study, distance is defined in terms of time rather than space. Time gaps are a function of spatial distance gaps and the follower's speed: because the LCV is concerned with having sufficient time, which is influenced by current speed that can vary, time gaps are more generalizable representations (Bham, 2009).

Lane changes can be classified into mandatory (MLC) or discretionary (DLC) motivations (Yang and Koutsopoulos, 1996), which, generally speaking, differ regarding gap acceptance (Toledo et al., 2003; Choudhury and Farheen, 2005). MLC is motivated by the driver needing to leave the current lane, e.g., to enter the correct lane for a turn, to enter or exit a limitedaccess freeway, or to avoid a work zone or other obstacle. DLC is motivated by the wish to improve conditions, such as moving from behind a slower vehicle in order to maintain a desired speed. Studies show that gap acceptance depends on several additional factors, e.g., spatiotemporal positions, relative speed, and acceleration of surrounding vehicles (Toledo et al., 2003; Bham, 2009; Choudhury and Farheen, 2005), most of which are directly linked to the current traffic condition (Singh and Li, 2012).

\subsection{Lane change duration}

Duration measures the length of the lane change execution stage, which starts when the LCV initiates its intention to change lane by a movement toward the target lane, and ends when LCV has stabilized itself in the target lane. Duration has significant effect on simulation outputs. For example, the LCV adjusts acceleration according to its perception of the needed duration; the FV, also, adjusts acceleration when it notices the initiation of the LCV. Consequently, the acceleration of the LCV and FV may, in turn, affect duration (Toledo and Zohar, 2007). In application, CAVs can learn to perform lane changes in a human-like manner by controlling the duration, and they can respond more effectively to lane change behavior of human-driven vehicles (Bascunana, 1995).

Lane change duration research has found values ranging from 1-16 s (Hanowski, 2000; Salvucci and Liu, 2002; Moridpour et al, 2010), but few studies have fully examined the influencing variables. Despite duration's wide value range and its significance in lane change, most existing microsimulation tools neglect it; rather, lane change behavior is generally considered as an almost instantaneous event or given a constant time period (Toledo and Zohar, 
2007). Nonetheless, most duration studies have been based on driving simulator data. While simulator research is useful for many objectives, this shortcoming may distort the realism of the simulated driving experience and the fidelity of the data collected (Singh and Li, 2012; Toledo and Zohar, 2007).

\subsection{Impact of lane changes}

A lane change event inevitably impacts the FV. Ahmed et al. (1996) developed a forced merging model and found that in congested traffic where gaps meeting the minimum acceptable length are scarce, drivers change lanes either through the FV's courtesy yielding or through forcing the FV to slow down. Lane changing also impacts traffic flow. An abrupt lane change that results in the FV's hard braking or excessive acceleration contributes not only to emissions and wasted fuel but also to traffic waves, or stop-and-go oscillations (Sultan et al, 2002). Oscillations are problematic because they trigger a drop in road capacity and increase the risk of rear-end crashes (Coifman et al, 2005). Mauch and Cassidy (2002) found that oscillations tended to form near freeway interchanges, sites of frequent lane changing. These maneuvers have been considered to enlarge the capacity drop phenomenon (Srivastava and Geroliminis, 2013). Yet, despite the effect of lane change impact on traffic safety, its analysis has received less attention than lane change decision-making and execution.

\section{Data preparation}

\subsection{Shanghai naturalistic driving study}

Naturalistic driving research offers a complementary approach to existing methods (Regan et al, 2012). It is more controlled than field research, and its data is more representative of real traffic conditions in relation to simulator data. The data used in this study were collected in the Shanghai Naturalistic Driving Study (SH-NDS) jointly conducted by Tongji University, General Motors (GM), and the Virginia Tech Transportation Institute (VTTI). Five GM light vehicles equipped with SHRP2 NextGen Data Acquisition Systems (DAS) were used to collect real-world driving data. The DAS includes an interface box to collect vehicle Controller Area Network (CAN) data, an accelerometer for longitudinal and lateral acceleration, a radar system that measures range and range rate to the lead vehicle (LV) and vehicles in the adjacent lanes, a light meter, a temperature/humidity sensor, a GPS sensor for location, and four synchronized cameras which can be used to validate the sensor-based findings (Wang et al, 2019).

\subsection{Lane change events extraction}

This study focuses on lane changes conducted by LCVs in the lanes adjacent to the DASequipped NDS vehicles. That is, the NDS vehicle functions as the FV, providing the perspective of the FV when another vehicle changes lane into the gap in front. Based on the fundamental information (e.g., position, velocity and acceleration) of the LCV recorded by the NDS vehicle's radar, lane change gap acceptance, duration, and FV responses (e.g., braking, speed variation and other maneuvers) can be examined. A typical lane change scenario is illustrated in Fig. 1. 


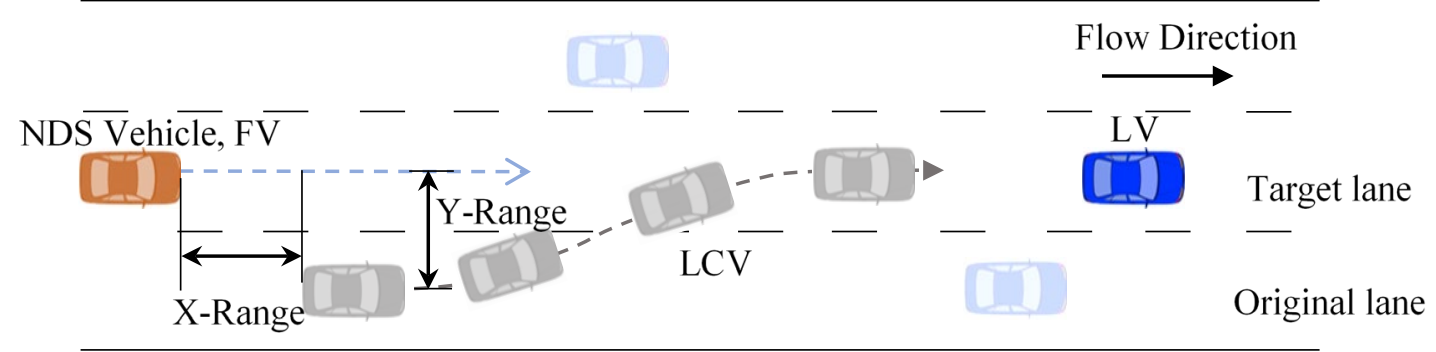

Fig. 1. Radar target's (LCV) position and motion in a typical lane change scenario.

As shown in Fig. 2, a vehicle directly in front of the NDS vehicle in the target lane is designated as T0. If the NDS's radar picks up a change in an adjacent vehicle's position in relation to the T0 position, the vehicle is recorded as intending to execute a lane change. In Fig. 2, the blue car far from the FV was initially T0, and then the white car moved into the NDS's lane: the white LCV is the new T0. The middle panel shows the lateral and longitudinal ranges of the vehicles in front of the FV, and the rightmost panel shows the Y-Range to vehicle T0 vs. time.
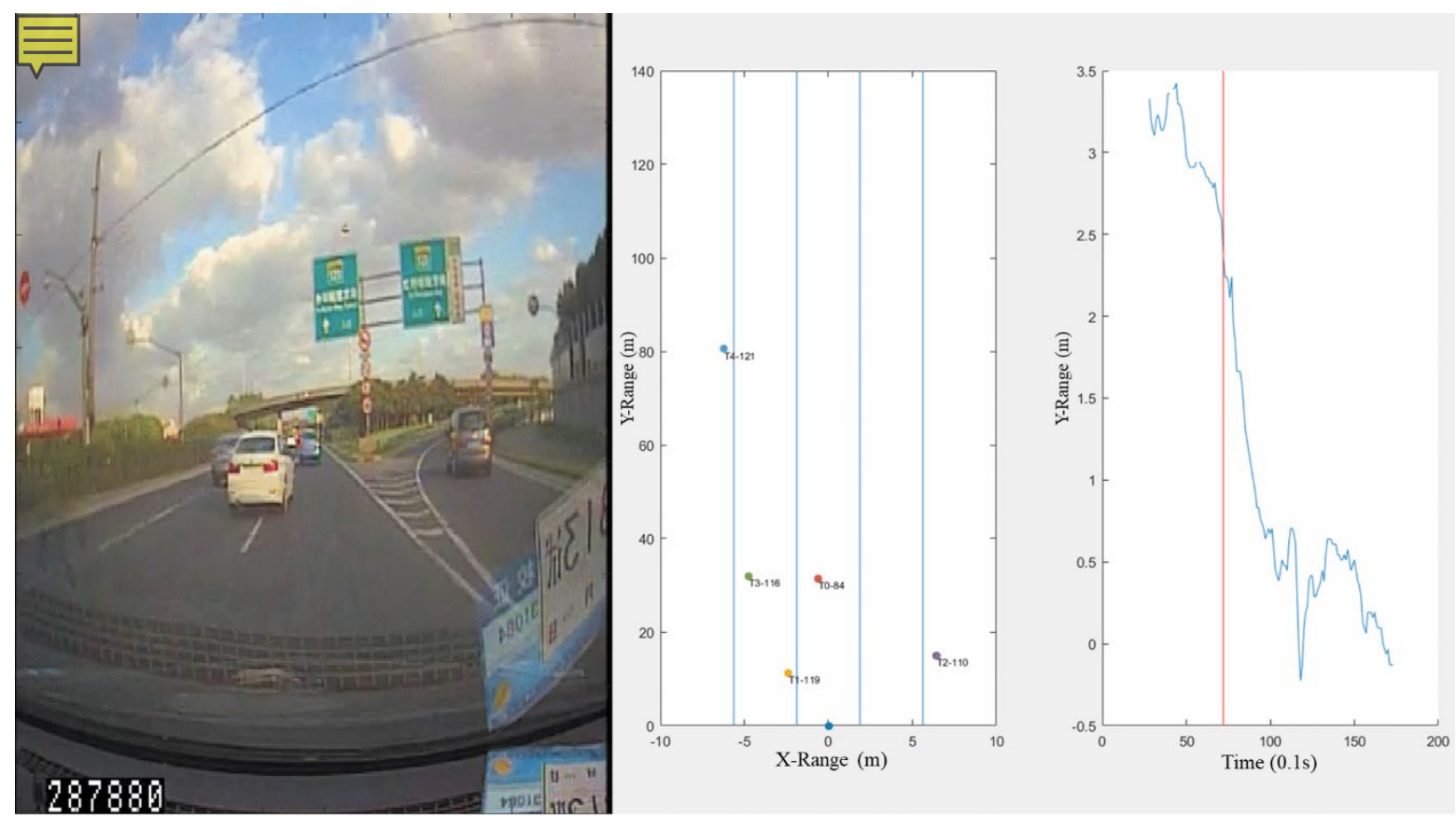

Fig. 2 Change in radar target $\mathrm{T} 0$.

An automatic extraction algorithm was developed to obtain lane change events, and the results were then manually validated by observing the videos from the forward roadway camera. To develop the automatic algorithm, an empirical analysis was conducted to identify threshold values for detecting lane change events from the NDS data. As part of the analysis, 500 random lane change events from 100 trips (5 lane change events for each trip) were manually observed to have a deep understanding of the distribution of some important variables, and extraction criteria were derived based on the thresholds of the several relevant variables.

For each of the abovementioned lane change events, we manually recorded the timestamps for the initiation, cross-lane maneuver and stabilization. All variables of the LCV at the specific 
1 timestamp were extracted from the SH-NDS database. We calculated the $10^{\text {th }}$ percentile 2 minimum lateral distance between the LCV and the FV (i.e., the Y-Range in Fig. 1, in an 3 ascending order) of all these events at the initiation point to better determine the threshold. The 4 results showed that the minimum of the $10^{\text {th }}$ percentile value was $2.19 \mathrm{~m}$. That is the Y-Range 5 should not be less than $2.2 \mathrm{~m}$ before the LCV initiates its movement. Similarly, the $90^{\text {th }}$ 6 percentile maximum Y-Range (in a descending order) of all these events at the stabilization 7 point was found to be $1.22 \mathrm{~m}$. It can be concluded that when the LCV stabilizes in the target 8 lane, the lateral distance between the LCV and the FV (i.e., Y-Range) should not be more than $9 \quad 1.2 \mathrm{~m}$. This means that the two thresholds represent the two different stages of a lane change 10 event.

Criterion 1: Y-Range (lateral distance) is more than $2.2 \mathrm{~m}$, to show the LCV is in the adjacent 14 lane and intend to initiate its movement toward the FV lane; Y-Range is less than $1.2 \mathrm{~m}$ to ensure the LCV is stable in the target lane. Together, these criteria guarantee the LCV changes its lane.

17 Criterion 2: Maximum lateral acceleration of FV is less than $0.07 \mathrm{~g}$, and lane offset is less than $1.0 \mathrm{~m}$. These criteria guarantee the FV does not move in a lateral direction.

19 Criterion 3: The critical condition is that the X-Range (longitudinal distance from LCV to FV) 20 should not be so large that the lane change has no effect on the FV. Based on the 500 observations and existing research (Bham, 2009; Herman and Weiss, 1961), this study defined the maximum $\mathrm{X}$-Range as $75 \mathrm{~m}$ to be appropriate to determine a pair of vehicles to be interacting.

Criterion 4: The speed of both FV and LCV should be more than $1 \mathrm{~m} / \mathrm{s}$. This criterion ensures that the two vehicles are always in motion.

The flowchart for the lane change event extraction algorithm is shown in Fig. 3. 


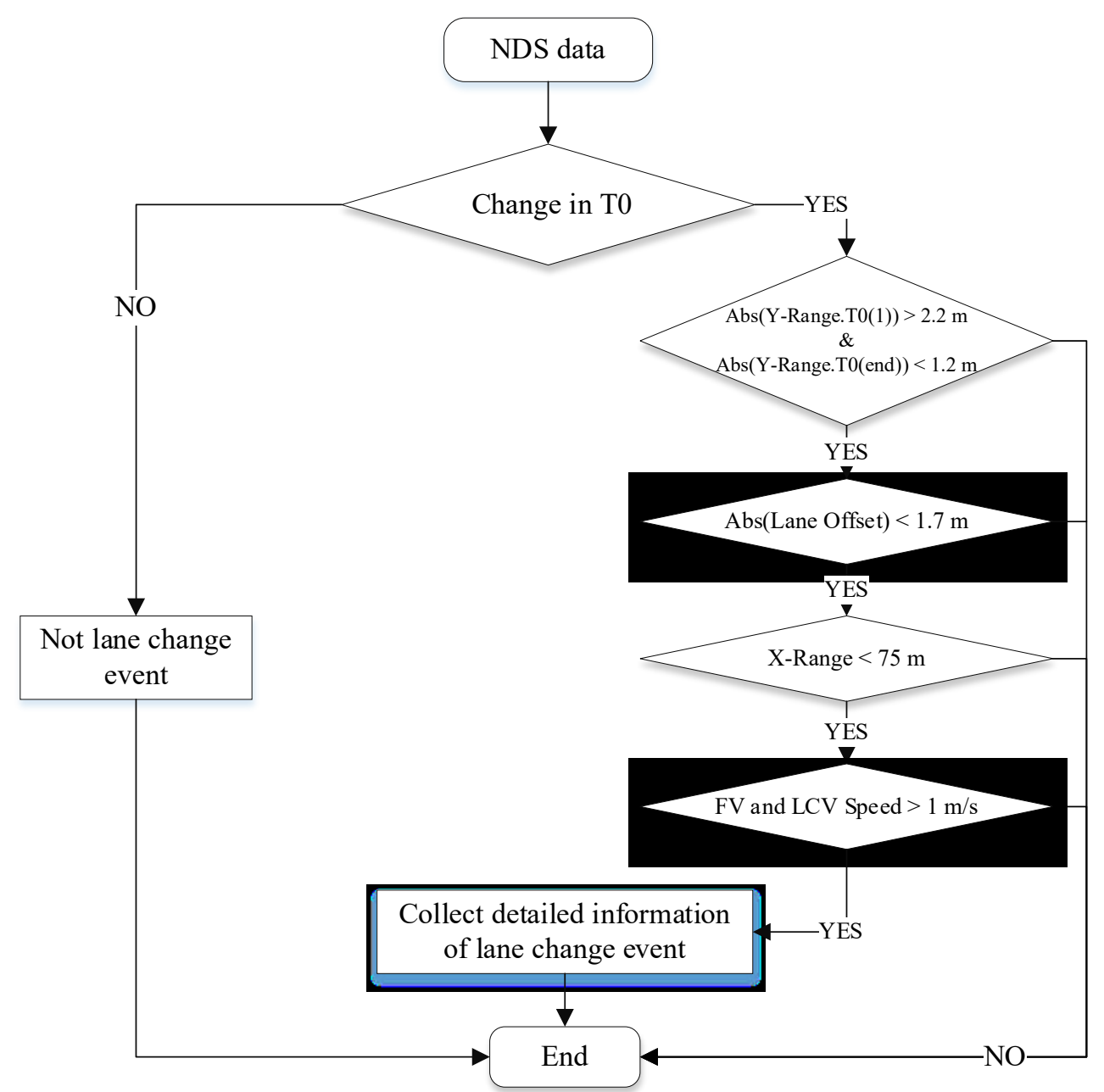

Fig. 3 Automatic extraction algorithm.

Fig. 4 illustrates the critical time points (A, B, and C) utilized in the DAS lane change data extraction process (Wang et al, 2019). At the onset of the lane change, the LCV's Y-Range (its lateral distance in relation to the FV) is about $3.5 \mathrm{~m}$, as the LCV is still in its own lane but has just initiated movement. The initiation point (A in Fig. 4) is defined as the first peak (i.e. the local maximum) of the Y-Range determined by a built-in function findpeaks of MATLAB, which is the nearest point to $3.75 \mathrm{~m}$, i.e., Y-Range is approximately equal to the common lane width in China when the LCV initiates its movement. When the LCV's Y-Range has decreased to less than the distance between the lane edge and the FV, it can be assumed that the LCV has crossed its lane. This is the cross-lane point (B in Fig. 4). At roughly $1.5 \mathrm{~s}$ from the cross-lane position, the LCV becomes T0. The Y-Range at this point, marked by the red vertical line, is small and approaching zero. The first zero value of Y-Range after the LCV becomes T0 is defined as the end of the lane change, or stabilization ( $\mathrm{C}$ in Fig. 4). From this point, it is considered that the LCV has completely stabilized its movement within the target lane. The lane change duration is defined as the time required for the LCV to travel from A to C, making the duration in Fig. 4 approximately $5.5 \mathrm{~s}$. 


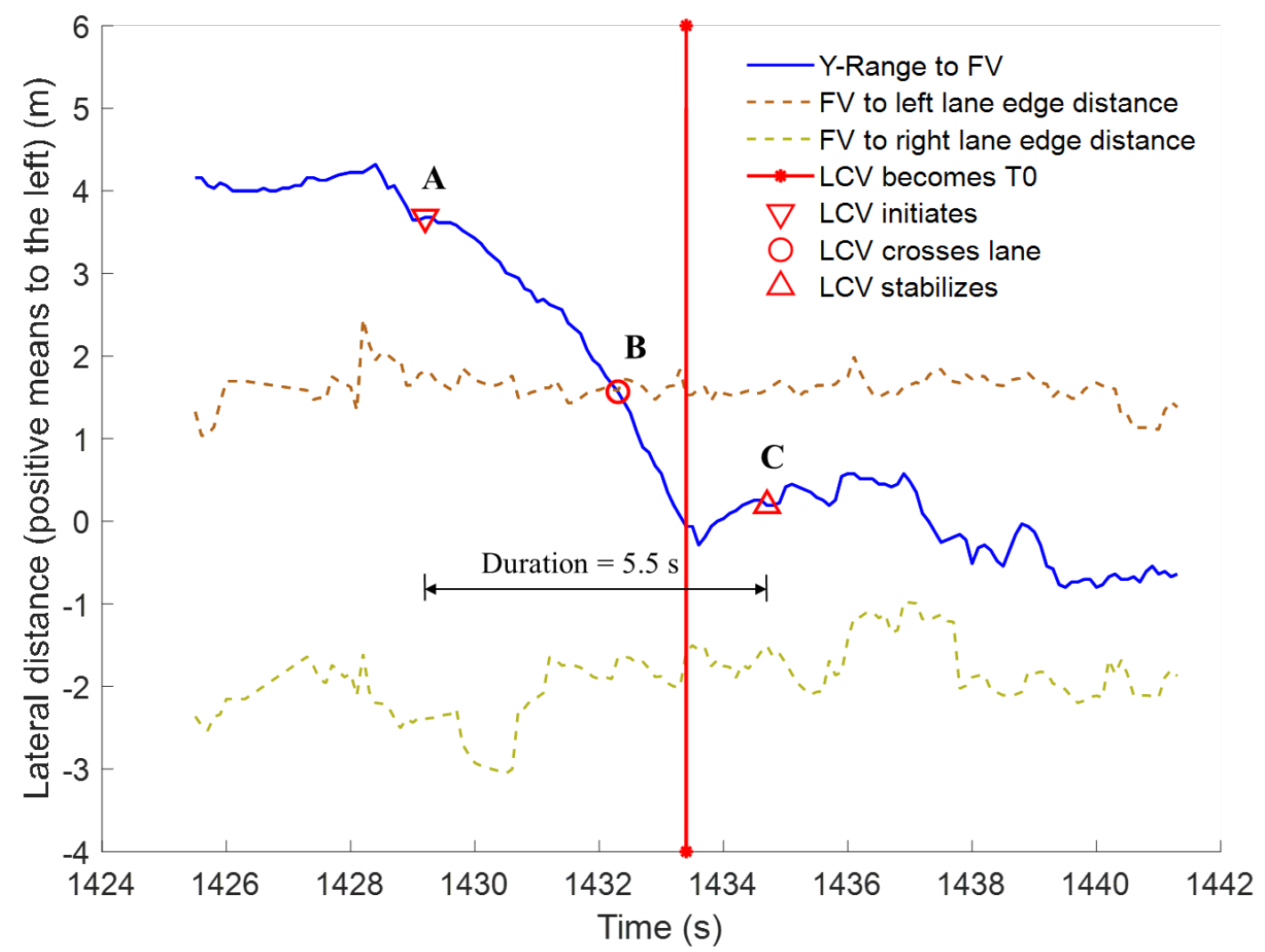

Fig. 4 Y-Range time points of lane change sequence: initiate, cross-lane and stabilize.

This study used 2,000 trips to extract lane change events from the SH-NDS database. The proposed automatic extraction algorithm was first employed to detect potential lane change events. The algorithm detected 6,672 such events from the 2,000 trips (about 600 hours of total trip duration). For each of the events, the corresponding video clips were manually observed to confirm whether these are actual lane change events. Results show that 1,333 events detected by the algorithm are not real lane change events (i.e. about a $20 \%$ false alarm rate). However, this study has only employed the remaining 5,339 lane change events for further analyses. Therefore, the false alarm rate did not influence the analysis.

In addition, we identified the reasons of this high false alarm rate as follows:

FV moves laterally but its lateral acceleration is too small. Meanwhile, many arterials and local roads contain no line marks or the lane lines are very unclear. In both conditions, Criterion 2 of the algorithm becomes invalid. That is: the FV moves in a lateral direction but the algorithm erroneously identifies a lane change maneuver of the LCV.

- If there is a road curve or when the FV makes a turn. If the FV passes the road curve or makes a turn, the Y-Range and the lane offset restrictions become incorrect. That means that road curve or the FV's drift may mislead Criterion 1 and Criterion 2.

- Lateral distance is small. In some cases, the LCV fails to enter the target lane but lateral distance between the LCV and the CV is less than $1.2 \mathrm{~m}$. Criterion 1 detects it as a lane change by mistake thus leads to false positive.

Nevertheless, the algorithm was extremely useful in identifying the video clips containing potential lane change events. If the algorithm was not employed, then we had to manually check all video clips from 2,000 trips resulting in a total duration of 600 hours which would have been difficult to accomplish. With the algorithm, we had to only go through about 28 
1 hours of videos $(6,672 \times 15 \mathrm{~s}=27.8$ hours $)$ resulting in a significant saving with respect to

2 time.

3

4

5

6

7

\subsection{Data description}

In this study, the fundamental data (e.g., position, velocity and acceleration) of lane change vehicles, lead vehicles and following vehicles were recorded by the NDS vehicles' (i.e., FV) radar and saved as CSV files. Other important information of lane change events, including roadway type, weather and light conditions, lane change motivation, turn signal usage and LCV and LV types, was identified by observing the forward-facing camera during the validation process.

The 5,339 lane change events occurred on three road types with different speed limits: 2,686 occurred on expressways with a speed limit range of $60 \mathrm{~km} / \mathrm{h}$ to $80 \mathrm{~km} / \mathrm{h}, 1,511$ on freeways with speed limits ranging from $80 \mathrm{~km} / \mathrm{h}$ to $120 \mathrm{~km} / \mathrm{h}$ and the remaining 1,142 occurred on other roads that include arterials and local roads with speed limits ranging from 30 $\mathrm{km} / \mathrm{h}$ to $80 \mathrm{~km} / \mathrm{h}$. Since the speed limits, roadway configurations and road complexity are quite different from each other, three separate analyses of gap acceptance, duration and impact of lane changes were conducted.

Lane change motivations were determined by watching the relevant video for each event. Following Yang and Koutsopoulos (1996), lane change motivations were classified as either mandatory or discretionary. Mandatory lane changes have three primary motivations: the first motivation is approaching an intersection in situations where the vehicle must change lanes to be in the correct lane to turn; the next motivation is entering or exiting a limited-access roadway, and the last motivation is avoiding a work zone or other obstacle. Motivations for discretionary lane changes include avoiding traveling behind a slow lead vehicle and changing to a fast or slow lane to maintain a desired speed. Lane changes without any clear motivation were considered discretionary in this study.

Based on the above description, a sample is a lane-changing event and then group them into roadway functional classifications and lane-changing motivation. Meanwhile, lead and lag gaps, duration and the following vehicles' response for each lane change event can be obtained by analyzing the SH-NDS data.

\section{Analysis of lane change gap acceptance}

Gap acceptance is the most essential determiner for drivers deciding whether to perform a lane change (Choudhury and Farheen, 2005). This section presents a descriptive analysis of lead and lag gaps, as well as a linear mixed-effects model aiming to discover the multiple variables that can affect gap acceptance.

\subsection{Analysis of lead and lag gaps}

Drivers assess the risk when they intend to execute lane changes. The risk factors to the LCV with respect to the intended LV and to the intended FV with respect to the LCV should be compared to an acceptable risk factor for every lane change. It is assumed that risk varies by time, e.g., the moment when complete entrance of the rear end of LCV to the target lane or the front end of LCV entering the target lane. Therefore, there is a need to do a sensitivity test to expound the potential difference.

To compare the results of lead and lag gaps for different lane change instances, time gaps 
1 in lane change initiation point and cross-lane point were calculated to analyze the deviation.

2 The results show that the cumulative distribution of lead and lag gaps by road type or by

3 motivation (i.e., mandatory or discretionary) at the initiation point is almost the same as that at

4 the cross-lane point, which indicates that the time gaps are insignificantly different before the

5 LCV enters the target lane completely.

6 Based on the above analysis, this study assumes that LCV drivers accept the gap when

7 they start to move into the target lane, i.e., the initiation point. Lead gap and lag gap refer

8 specifically to the time taken to traverse the longitudinal distance between the LCV and LV

9 and between LCV and FV once LCV initiates its lateral movement. A descriptive analysis of

10 lead and lag gaps on different roads and for different motivations was conducted to ascertain

11 the features of gap acceptance.

Table 1

Descriptive statistics of lead and lag gap.

\begin{tabular}{ccccccc}
\hline \multirow{2}{*}{ Statistics } & Gaps & \multicolumn{3}{c}{ Road Type } & \multicolumn{2}{c}{ Motivation } \\
\cline { 2 - 7 } & & $\begin{array}{c}\text { Arterials and } \\
\text { Local Roads }\end{array}$ & Expressways & Freeways & MLC & DLC \\
\hline \multirow{2}{*}{ Sample Size } & Lead & 758 & 2,056 & 1,018 & 781 & 3,051 \\
\cline { 2 - 7 } & Lag & 1,142 & 2,686 & 1,511 & 1,093 & 4,246 \\
\hline \multirow{2}{*}{ Min (unit: s) } & Lead & 0.16 & 0.14 & 0.13 & 0.13 & 0.14 \\
\cline { 2 - 7 } & Lag & 0.18 & 0.15 & 0.14 & 0.14 & 0.15 \\
\hline \multirow{2}{*}{ Max (s) } & Lead & 5.98 & 5.98 & 5.96 & 5.98 & 5.96 \\
\cline { 2 - 7 } & Lag & 5.79 & 5.75 & 5.56 & 5.79 & 5.75 \\
\hline \multirow{2}{*}{ Mean (s) } & Lead & 1.83 & 1.48 & 1.42 & 1.76 & 1.47 \\
\cline { 2 - 7 } & Lag & 1.80 & 1.37 & 1.26 & 1.78 & 1.34 \\
\hline \multirow{2}{*}{ Median (s) } & Lead & 1.60 & 1.25 & 1.18 & 1.50 & 1.25 \\
\cline { 2 - 7 } & Lag & 1.56 & 1.18 & 1.08 & 1.56 & 1.15 \\
\hline \multirow{2}{*}{ Std. Dev (s) } & Lead & 1.15 & 0.97 & 0.93 & 1.16 & 0.96 \\
\cline { 2 - 7 } & Lag & 1.04 & 0.84 & 0.97 & 1.03 & 0.84 \\
\hline
\end{tabular}

15

As shown in Table 1, the mean and median values of lead and lag gap on arterials and local roads are larger than those on expressways and freeways, while the differences between expressways and freeways are not significant ( $p$-value $=0.156$ ). For all road types, the lead gap's mean and median values, as well as its maximum values, are comparatively greater than those of the lag gap. These results indicate that LCV drivers tend to maintain a larger space behind the LV, while they are more accepting of smaller headways when interacting with the FV. A possible explanation is that because LCV drivers depend on mirrors to determine the lag gap, their perception of that gap may not be as reliable as it is for the lead gap.

It is evident that drivers need a larger time gap to execute an MLC, as the mean and median values associated with MLC are higher than those of DLC (significant difference, $p$-value = 0.041). This difference suggests that drivers are more cautious when they perform an MLC. The result is consistent with Zhang and Kovvali (2007) who found that MLC maneuvers require larger gaps than DLC maneuvers. Since an MLC means that drivers are forced to insert their vehicles into the target lane, they must make adjustments in order to create a sufficient space. In contrast, a DLC depends on the driver's more spontaneous desire to drive at faster speeds, which may lead to more rapid maneuvers. Additionally, FV drivers often reduce their acceleration to ensure that a free gap of sufficient length is created during the next few seconds in MLC. However, in DLC, FV drivers may not provide courtesy as LCV drivers just want to 
1 pursue for higher speed or space. A combination of the larger longitudinal distance and the slower FV, therefore, contribute to shorter time gaps in MLC than in DLC.

The cumulative percentage curves of lead and lag gap are shown in Fig. 5. Similar to the descriptive statistics presented in Table 1, lead and lag gap both have a lower percentage of risky small gaps (time gaps a 3 s) on arterials and local roads than on expressways and freeways. Since the operational environment of arterials and local roads is complex with its numerous bus stops, high signal density and unexpected pedestrians in the outermost lane, drivers travelling these roads tend to perform lane changes with a larger safety margin. Additionally, the lower speed limit makes lane changes less urgent. DLC occupies a dominant position in smaller gaps, which is consistent with the analysis above.
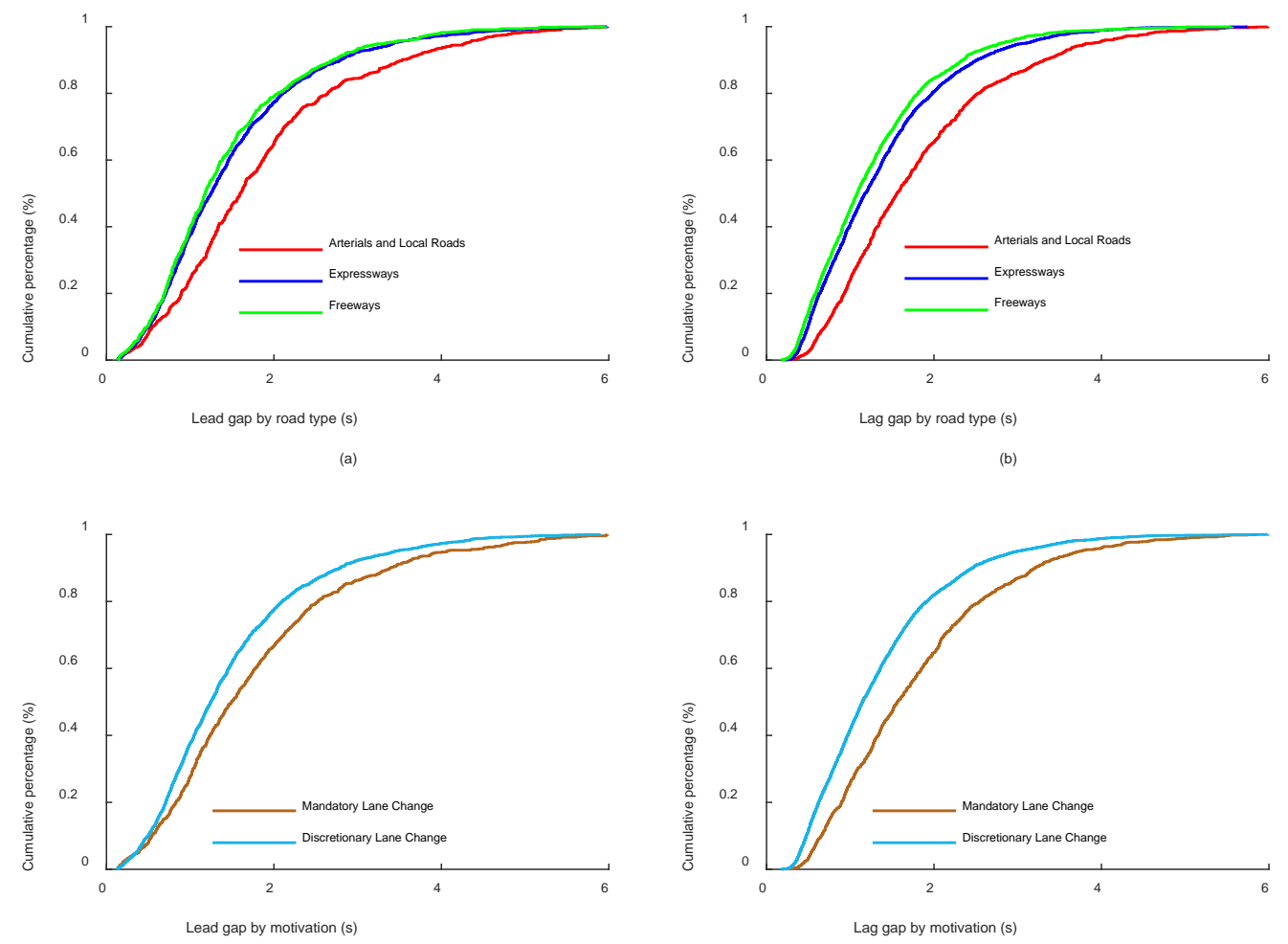

Fig. 5 Lead and lag gap cumulative percentage: (a), (b) by road type; (c), (d) by motivation.

\subsection{Multi-level model for lead and lag gaps}

The aim is to develop a statistical model for identifying factors affecting lead or lag gaps. Data on lane change events are hierarchical, as shown in Fig. 6. For instance, lead gaps are nested within lane change motivations (MLC and DLC) and lane change motivations are further nested within road type. Moreover, lead gaps under MLC may have some similarities i.e., within-cluster correlation. On the other hand, there might be variation between lead gaps from different lane change motivations and/or different road types, i.e., between-cluster variation. Therefore, a statistical model is needed to jointly control both within- and betweencluster variations (Deligianni et al, 2017). 




Fig. 6 Multi-level model for lane change events (lead gap example).

A multilevel mixed-effects linear regression model fills this need. Specifically, a threelevel random-intercept and random-coefficient model is most suitable for lane change gap acceptance because it allows the dependent variable of gap acceptance to have different characteristics for the same roadway and within the same motivation. Additionally, the model examines the variation of gap acceptance characteristics on different roadways, and the variation of MLC or DLC on the same roadway. A three-level random-effects linear regression model (STATA Reference Manual, 2013) can be developed for a single explanatory variable $(x)$ as:

Lane change - event-level (level-1):



Lane change - motivation-level (level-2):

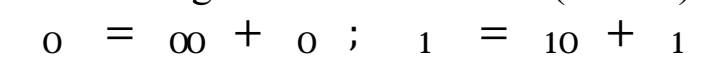

Lane change - roadway-level (level-3):

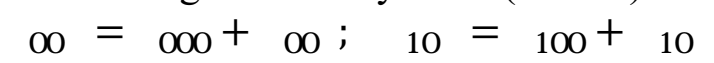

The composite equation can be expressed as:

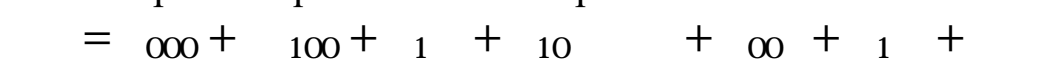

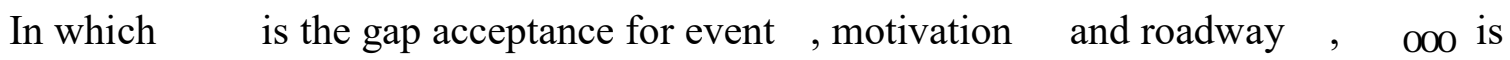

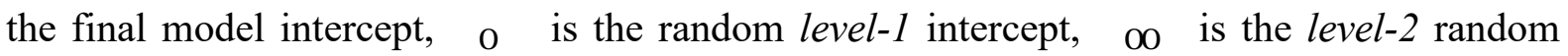
intercept, ' OHI is the level-1 residual, $\Xi_{\text {annc: }}$ is the fixed slope coefficient for explanatory


slope coefficient for $x$. All random components are assumed to follow a normal distribution with a mean of zero and a constant standard deviation. Eq. (4) represents a three-level randomeffects linear regression model for a single explanatory vehicle but can be extended for multiple explanatory variables.

The independent variables used in gap acceptance are listed in Table 2. These explanatory variables for lane change behavior can be classified into some common groups based on previous studies:

, Neighborhood variables - The surroundings of LCV strongly affect the behavior. Most importantly, the presence of other vehicles in the target lane and their actions directly influence LCV drivers' decisions. Gap acceptance decision-making depends on the speed of the LCV with respect to the vehicles surrounding it. Therefore, both LCV-to-LV and 
LCV-to-FV relative speed and vehicles' acceleration (i.e., LCV, LV and FV acceleration) were extracted to capture gap acceptance decision-making process (Toledo et al., 2003; Hill and Elefteriadou, 2013; Hou et al., 2015; Lee et al., 2016).

- Environmental variables - Weather and light conditions may strongly affect the lane change behavior and gap acceptance (Chen et al., 2018; Dissanayake et al., 2002). Roadway pavement condition (i.e., dry or wet) depends on weather and has a significant impact on the performance of vehicle dynamics. In addition, drivers' reaction time in bad weather may be longer leading to larger gaps. Similar to weather condition, drivers are prone to be influenced by lighting conditions. Another important environmental variable is traffic safety. Previous studies have shown that traffic flow oscillations are often accompanied by lane change maneuvers. More specifically, lane change frequency and gap acceptance vary with different traffic density (Singh and Li, 2012; Cooper et al., 2007).

, Vehicle type- Despite their smaller proportion of vehicular traffic, heavy vehicles are known for their important impacts on traffic flow (Al-Kaisy et al., 2002). Heavy vehicles may impose some physical and psychological effects on surrounding traffic due to their physical (e.g., length and weight) and operational (e.g., acceleration, deceleration, and maneuverability) characteristics (Al-Kaisy and Jung, 2004). Therefore, LCV type was observed and used as a key factor in gap acceptance model.

Parameters of all components were estimated using Stata ${ }^{\circledR} 13$ (STATA Reference Manual, 2013). As shown in Table 2, the three-level mixed-effects linear regression model provides a good fit to the data for lead and lag gaps. All variables listed in Table 2 are significant at the $95 \%$ confidence level ( $\mathrm{p}$-value $<0.05$ is significant).

Traffic density was identified through observing the forward roadway camera video recording, and verified by the averaged travel speed of FV. In this study, the low-density state is defined as a large inter-vehicle spacing with less than three vehicles traveling in front of NDS vehicle while traffic moves more than two-thirds of the speed limit. The mediumdensity state is defined as a condition when travelling speed is one to two-thirds of the speed limit with three to five vehicles in front of NDS vehicle, and high density is indicated by slow speeds (i.e. less than one-third of the speed limit) with more than five vehicles in proximity.

The variable high traffic density (crowded traffic with slow travel speed) shows a positive coefficient, indicating that lead gaps increased in high density traffic. The heavy traffic encourages drivers be more cautious, as improper lane change timing will increase the risk of crashes. This result is consistent with Bham (2009), who investigated the gap acceptance behavior in lane-changing situation on freeways using the Next Generation Simulation (NGSIM) data. In the NGSIM project, cameras were used to record video images of vehicles on a road from the top of the tall buildings and yields trajectory datasets which were used to analyze driving behavior. In Bham (2009), gaps of mandatory lane changes are larger for congested conditions compared to uncongested conditions.

Bad weather (e.g., rainy) and poor light conditions (e.g., nighttime) led to larger lead gaps, and also to larger lag gaps. LCV drivers need more time in these conditions to adjust the vehicle's movement and react to urgent situations. The $L C V$ type in the lead-gap model significantly affects lead gaps. Presence of heavy vehicles as lane change vehicles tend to increase accepted lead gaps for both MLC and DLC maneuvers, which can be attributed to drivers' abundance of caution because of heavy vehicles' length, size, weight and limitations 
1 in their maneuverability (Kayvan et al., 2011). This finding is the same as the result in Zhang 2 and Kovvali (2007), who conducted a detailed analysis of lane change gap-acceptance based

3 on the NGSIM data.

Table 2

Three-level mixed-effects linear regression models for lead and lag gap acceptance.

Dependent variable

Lead Gap

Lag Gap

\begin{tabular}{|c|c|c|c|c|c|c|}
\hline Fixed effect & Coefficient & t-stat & $\operatorname{Pr}>|t|$ & Coefficient & t-stat & $\operatorname{Pr}>|t|$ \\
\hline $\begin{array}{c}\text { Traffic density: } \\
\text { High } \\
\text { Medium } \\
\text { Low (Reference) }\end{array}$ & $\begin{array}{l}0.3625 \\
0.1217\end{array}$ & $\begin{array}{l}7.34 \\
2.72\end{array}$ & $\begin{array}{c}<0.001 \\
0.007\end{array}$ & $\begin{array}{l}0.4502 \\
0.0630\end{array}$ & $\begin{array}{c}11.86 \\
1.83\end{array}$ & $\begin{array}{c}<0.001 \\
0.067\end{array}$ \\
\hline $\begin{array}{c}\text { Weather condition: } \\
\text { Rainy } \\
\text { Sunny (Reference) }\end{array}$ & 0.1087 & 1.94 & 0.053 & 0.1183 & 2.71 & 0.007 \\
\hline $\begin{array}{c}\text { Light condition: } \\
\text { Nighttime } \\
\text { Daytime (Reference) }\end{array}$ & 0.1925 & 4.84 & $<0.001$ & 0.0805 & 2.60 & 0.009 \\
\hline $\begin{array}{l}\text { LCV type: } \\
\text { Heavy vehicle } \\
\text { Light vehicle (Reference) }\end{array}$ & 0.3219 & 3.55 & $<0.001$ & - & - & - \\
\hline LCV acceleration & 0.1222 & 5.99 & $<0.001$ & -0.0731 & -4.60 & $<0.001$ \\
\hline 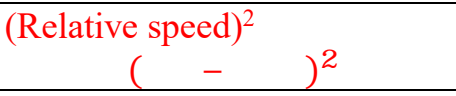 & 0.0060 & 4.53 & $<0.001$ & 0.0021 & 1.90 & 0.057 \\
\hline LV acceleration & -0.0609 & -3.60 & $<0.001$ & - & - & - \\
\hline 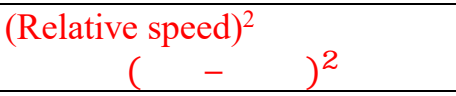 & 0.0137 & 7.72 & $<0.001$ & 0.0041 & 2.94 & 0.003 \\
\hline FV acceleration & -0.0504 & -2.29 & 0.022 & 0.0428 & 2.52 & 0.012 \\
\hline Intercept & 1.2858 & 11.94 & $<0.001$ & 1.3207 & 11.35 & $<0.001$ \\
\hline Random effect parameters & Estimate & & & Estimate & & \\
\hline $\begin{array}{l}\text { Variance of LC type } \\
\text { Reference: MLC }\end{array}$ & $3.62 \mathrm{e}-08$ & & & $9.35 \mathrm{e}-06$ & & \\
\hline $\begin{array}{l}\text { Variance of road type } \\
\text { Reference: Arterials and Local } \\
\text { Roads }\end{array}$ & 0.2387 & & & 0.2718 & & \\
\hline Variance of Residual & 1.0415 & & & 0.8935 & & \\
\hline LR test vs. linear regression & \multicolumn{3}{|c|}{$3^{76}=136.47$, Prob $>z^{7 \leftarrow}=0.0000$} & \multicolumn{3}{|c|}{$3^{76}=315.91$, Prob $>z^{76}=0.0000$} \\
\hline $\begin{array}{l}\text { Statistics } \\
\text { Number of observations } \\
\text { Number of groups } \\
\text { Log-likelihood }\end{array}$ & $\begin{array}{c}3735 \\
6 \\
-5461.1686 \\
\end{array}$ & & & $\begin{array}{c}4473 \\
6 \\
-5854.9650 \\
\end{array}$ & & \\
\hline
\end{tabular}

Both the square of the relative speed (unit: $\mathrm{m}^{2} / \mathrm{s}^{2}$ ) and acceleration (unit: $\mathrm{m} / \mathrm{s}^{2}$ ) affected the gap acceptance. LCV acceleration had different effect on lead gaps and lag gaps, i.e., the

10 faster the LCV, the larger lead gap and smaller lag gap it needs. The results of the lead gap 11 model show that acceptable lead gaps increased with the increase in the square of LCV-to-LV 12 relative speed, and decreased with the increase in LV acceleration. This can be understood as 13 LCV drivers preferring to accept a larger gap when the LV accelerates in order to create a 14 suitable space between the two vehicles. This result is consistent with Gurupackiam and Jr's 15 (2002) research, which found that in non-recurrent congestion, drivers, on the average, 
1 accepted smaller gaps. An increase in LCV-to-FV relative speed and an increase in FV acceleration, however, reduced the lead gap. A possible reason is that faster relative speed of the FV increases the risk of rear-end crash, which may encourage the LCV driver to accept a smaller lead gap.

Results of the lag model show that LV acceleration have no significant effect. In contrast to lead model in Table 2, a slower LV and a faster FV pressure the LCV driver to accept a larger lag gap. One reasonable explanation is that LCV drivers attempt to increase the size of the gap when noticing the LV is slowing down and the FV is closing in on them, as they have less perception of the rear space.

\subsection{Discussion on gap acceptance}

Generally speaking, the drivers of lane-changing vehicles do not reject gaps that are larger than the accepted gaps, which is known as consistent behavior (Bham, 2009). However, drivers, sometimes, reject an accepted gap due to various reasons: (i) avoiding collisions between the LV and the FV in the target lane, (ii) occurring an emergency situation inside the vehicle, (iii) failing to reach the intended destination, (iv) distracted driving. A minimum acceptable gap from a population, however, cannot be considered a critical gap as "it would only represent the gap acceptance behavior of an aggressive driver and would not represent the minimum acceptable gap of the driver population" (Bham, 2009). The rejected gaps, therefore, may or may not be less than the accepted gaps.

This study develops a lane change gap-acceptance model based on the observed gaps (i.e., the time gaps when LCV drivers began to move into the target lane) instead of the minimum acceptable gaps. The reasons for this are briefly explained below:

First, either for mandatory lane changes or discretionary lane changes, LCV drivers' unobserved lane change intentions cannot be inferred from observed behaviors, i.e., from the perspective of the FV, all we can utilize are the data from the radar system and the forwardfacing roadway camera. In fact, the real intentions of LCV drivers can only be approached by using specific instruments, including in-vehicle cameras, eye tracker, electroencephalograph, etc. For instance, in Olsen et al. (2002), the lane change initiation point was identified using a criterion that "driver returns gaze to the forward view after looking in mirrors or looking directly toward the side or rear". According to some previous studies (Salvucci et al., 2007; Toledo and Zohar, 2007), it is appropriate to define lane change initiation as the vehicle starts moving toward another lane and continues, without reversal, through to the target lane.

Secondly, as for mandatory lane changes, the minimum acceptable gaps depend on various factors. For example, the distance to the exit location on freeways affect drivers' lane change decision-making process. In addition, traffic density in the intersection areas cannot be ignored when drivers perform lane changes on arterials and local roads. This study combines all lane changes related to mandatory maneuvers as MLC. The minimum acceptable gaps, therefore, are hard to extract for each MLC event for different scenarios.

As for discretionary lane changes, we attempted to extract time gaps data five to ten seconds prior to a lane change, during which may include the maximum rejected gaps, or minimum acceptable gaps (Bham, 2009). However, a large amount of inconsistent gap acceptance behavior was observed in DLC events, i.e., drivers accepted shorter gaps rather than subsequent safer gaps. Additionally, considering the limited traffic resources, drivers executing a DLC were more aggressive with significant acceleration behavior to seek a better driving 
1 environment, which caused the DLC acceptable gap was always lower than the critical gap.

2 This can also attribute to the shorter gap acceptance in a DLC, which, in fact, has been

3 discussed in the above section.

4 To understand the drivers' lane change decision-making mechanism, this study also 5 attempted to examine LCV turn signal activation. According to the authors' knowledge, turn 6 signal could be used to locate a lane change as an auxiliary criterion. However, due to the fact 7 that only half of Chinese drivers use a turn signal when performing lane changes in this study, 8 we cannot offer insight into lane changes by analyzing turn signal usage. In addition, Olsen et 9 al. (2002) pointed out that turn signal activation cannot be relied upon as the initiation point of 10 a lane change since it is not present in all lane changes and does not always represent the initiation point of the maneuver.

Considering the difficulty in extracting the minimum acceptable gaps from the SH-NDS database, we decide to use the time gaps in the defined initiation point (i.e., point A in Fig. 4 ) as the minimum acceptable gaps for each lane change event. Although the values may not be very precise, the initial lateral movement of LCV indicates that the necessity, desirability and safety of lane changes at that moment have met the needs of LCV drivers and they can approximately capture the meaning of gap acceptance (Toledo et al., 2003).

\section{Lane change duration}

Duration is one of the most important parameters for research in lane change maneuvers. In the 5,339 events studied, the duration varies from $0.7 \mathrm{~s}$ to $16.1 \mathrm{~s}$ with the mean of $3.81 \mathrm{~s}$ and the standard deviation of $2.03 \mathrm{~s}$. Like gap acceptance, there are many variables that affect the duration of a lane change. As with gap acceptance, a three-level mixed-effects linear regression model was developed to explore the variables affecting lane change duration, and the same set of explanatory variables was employed. However, because initial results showed there was no significant relationship between lane change duration and motivation ( $p$-value $=0.432$ ), motivation-level was removed in the duration model. The resulting two-level model, which only considers roadway-level, is presented in Table 3.

\section{Table 3}

Two-level mixed-effects linear regression models for lane change duration on different road types.

\section{Dependent variable}

\section{Lane Change Duration}

\begin{tabular}{|c|c|c|c|}
\hline Fixed effect & Coefficient & t-stat & $\operatorname{Pr}>|t|$ \\
\hline $\begin{array}{c}\text { Traffic density: } \\
\text { High } \\
\text { Medium } \\
\text { Low (Reference) }\end{array}$ & $\begin{array}{c}0.2822 \\
-\end{array}$ & $\begin{array}{c}3.93 \\
-\end{array}$ & $\begin{array}{c}<\mathbf{0 . 0 0 1} \\
-\end{array}$ \\
\hline $\begin{array}{c}\text { Weather condition: } \\
\text { Rainy } \\
\text { Sunny (Reference) }\end{array}$ & 0.0088 & 0.08 & 0.934 \\
\hline $\begin{array}{c}\text { Light condition: } \\
\text { Nighttime } \\
\text { Daytime (Reference) }\end{array}$ & 0.1152 & 1.53 & 0.127 \\
\hline $\begin{array}{l}\text { LCV type: } \\
\text { Heavy vehicle } \\
\text { Light vehicle (Reference) }\end{array}$ & -0.0667 & -0.39 & 0.697 \\
\hline LCV acceleration & -0.0767 & -1.95 & 0.051 \\
\hline
\end{tabular}




\begin{tabular}{|c|c|c|c|}
\hline Lead gap & 0.0295 & 0.96 & 0.336 \\
\hline Relative speed $\left(a_{3 a^{\circ}}^{N} \widetilde{a}_{32 \pi}\right)$ & -0.0325 & -2.04 & 0.042 \\
\hline LV acceleration & 0.0286 & 0.89 & 0.373 \\
\hline Lag gap & -0.3075 & -8.23 & $<0.001$ \\
\hline 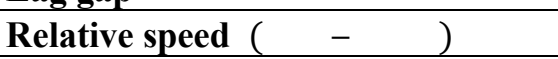 & 0.1744 & 10.11 & $<0.001$ \\
\hline FV acceleration & 0.1191 & 2.80 & 0.005 \\
\hline Intercept & 4.2641 & 43.79 & $<0.001$ \\
\hline \multicolumn{4}{|l|}{ Random effect parameters } \\
\hline $\begin{array}{l}\text { Variance of LC type } \\
\text { Reference: MLC }\end{array}$ & Insignificant & & \\
\hline $\begin{array}{l}\text { Variance of road type } \\
\text { Reference: Arterials and Local Roads }\end{array}$ & 0.0971 & & \\
\hline Variance of Residual & 1.9639 & & \\
\hline LR test vs. linear regression & \multicolumn{3}{|c|}{$z^{16}=2.60$, Prob $>z^{16}=0.0535$} \\
\hline Statistics & & & \\
\hline Number of observations & 3735 & & \\
\hline Number of groups & 3 & & \\
\hline Log-likelihood & -7821.3207 & & \\
\hline
\end{tabular}

1

As shown in Table 3, roadway type has a significant impact on lane change duration. Results also show that duration is increased by high traffic density and nighttime conditions. It can be easily understood that congested traffic flow and poor lighting make lane changing a more difficult maneuver, as the conditions require needs more time for drivers to prepare, execute and stabilize. Relative speed to FV and LV have different effects on duration, i.e., the faster the LV, the less time the LCV driver needs to complete the lane change; while a faster FV makes the duration longer. It is reasonable that when approaching the LV, or when the FV is closing, the driver of the LCV completes a lane change maneuver in a short time in order to guarantee safety. FV acceleration has the same effect on duration as LCV-to-FV relative speed, but increases in LCV acceleration and lag gap reduce lane change duration, which indicates that an LCV with speed and space advantage needs less time for execution. The other variables, however, of weather, LCV type, LV acceleration, and lead gap, are insignificant in the twolevel model.

The above results are consistent with the study of Toledo and Zohar (2007). Their lane change duration model shows that duration increases when traffic density is higher since it becomes more difficult and riskier to undertake the lane-changing action. Similarly, Kayvan et al. (2011) used NGSIM data to model lane change and came to the same conclusion. The lane change duration is longer when LCV-to-FV relative speed increases and LCV-to-LV relative speed decreases. Toledo and Zohar (2007) concluded that in order to make duration model be more useful, additional models need to be developed that describe the behavior of the LCV and the response of other vehicles around it during the lane change (e.g., in terms of speed and acceleration). This is exactly one of the reasons that lane change impact and the interaction between LCV and FV are analyzed in next section.

\section{Lane change impact on the following vehicle}

\subsection{Following vehicle speed change response}

By manually analyzing 1,000 lane change events, it was found that LCV drivers often completed lane changes by forcing the FV to slow down. This most common response is shown in Fig. 6(a), where FV speed decreases from the point when the LCV initiates a lane change 
1 until it stabilizes in the target lane. In contrast, however, a certain number of FV drivers maintain their current speed (see Fig. 6(b)) or even accelerate in response to the LCV, seemingly out of discourtesy or intolerance (see Fig. 6(c)).
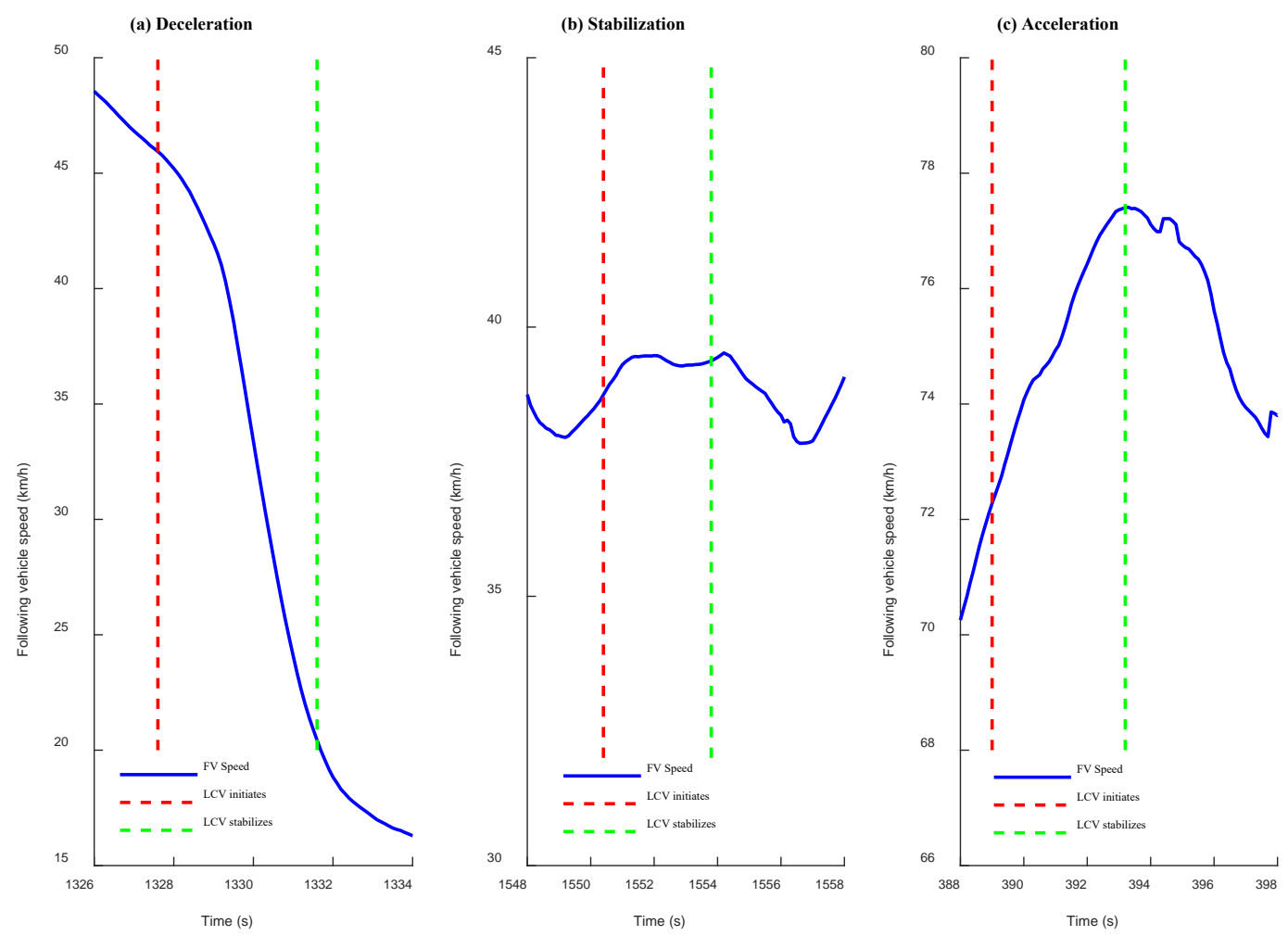

5

Fig. 6 Possible FV responses: deceleration, stabilization, acceleration.

Speed change rate is introduced as a practical indicator of the FV driver's response, which is defined in Eq. (5):

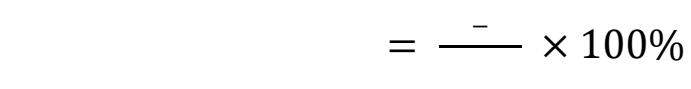

Where, $\|_{D}$ and $\|_{A}$ refer to the speed of the FV at the moments when the LCV initiates and stabilizes, respectively. Rates of speed change higher than $20 \%$ (absolute value) define abrupt deceleration or acceleration, and only these abrupt, or unstable, change rates are indicative of an adverse impact on the FV. To capture lane change impact more precisely, this study only considers those events with a speed change rate higher than $5 \%$, as they could directly affect the maneuvers of FV. Consequently, 3,101 of the 5,339 lane change events were used to analyze the acceleration and deceleration behaviors of the FV.

In most of the 3,101 events, lane changes have limited influence on the FV, which can be confirmed by the larger proportion of small speed change rates $(-10 \%,-5 \%)$ toward the centers of Fig. 7(a) and 7(b); but, like the LCV, the FV responds to different variables. As shown in the far ends of Fig. 7(a), FVs accelerate and decelerate more abruptly on arterials and local roads than on expressways or freeways, which can likely be attributed to arterials and local roads having more interrupted traffic flow and more challenging interactions with buses, bicycles, pedestrians and other road users. As can be seen at the far ends of Fig. 7(b), mandatory lane changing triggers more unstable reactions from the FV than does discretionary lane changing. 
1 As MLC always implies necessity, these LCV drivers may pay less attention to the rear space, 2 leading to more urgent interactions with the FV.

3 It is to a certain degree surprising that some FV drivers accelerate when the LCV executes 4 a lane change. It may be that the space between the FV and LCV is perceived by the FV as 5 sufficient, and the FV determines that acceleration will not lead to serious consequences. In 6 some cases, however, the FV appears to accelerate in an attempt to close the gap between itself 7 and the LCV in order to prevent the lane change, but fails. Video analysis shows that as many 8 as $20 \%$ of FVs respond with an acceleration rate of more than $10 \%$. This percentage, 9 significantly higher than the percentage for U.S. drivers, substantiates results from other studies 10 that find Chinese drivers to be more aggressive (Wang et al, 2016).

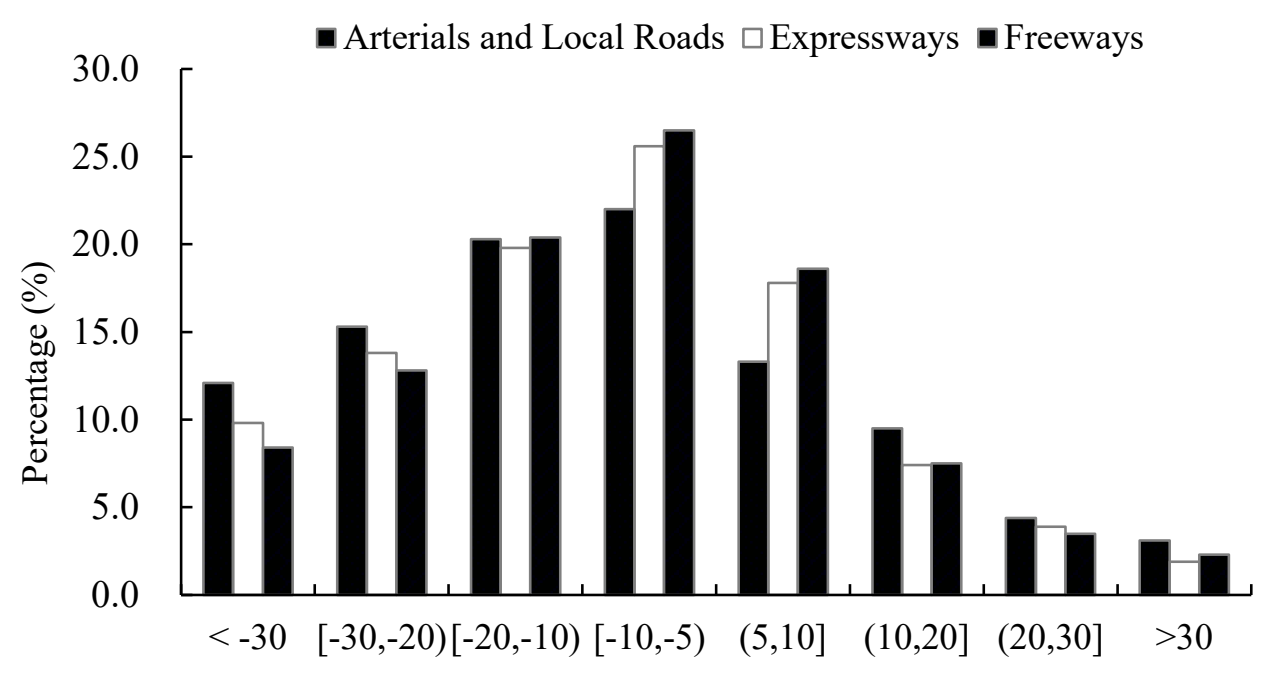

(a) Speed change rate $(\%)$



(b) Speed change rate for different motivation (\%)

Fig. 7 Speed change rate of following vehicles on different road types and for different motivations. 
The impact of lane change on the FV can also be analyzed through FV drivers' braking response. The DAS in NDS vehicles records the braking timestamp for the brake pedal position variable. As shown in Fig. 8, among all 5,339 events, 44.0\% of FV drivers brake when the LCV initiates its movement but before it crosses the lane line, while only $14.1 \%$ brake after the LCV crosses into the target lane, and $41.9 \%$ do not brake at all. The FV's braking response is consistent with its relatively small speed change, and supports the observation that a substantial number of lane changes have little impact on the FV. That $44.0 \%$ of drivers brake between the LCV initiation and cross-lane point offers insight into improving ADAS. For example, most forward collision warning (FCW) systems alert a driver to perform maneuvers to avoid a collision with the closest vehicle directly ahead. This study demonstrates, however, the value of improving FCW functions to perceive vehicles, such as an LCV, in adjacent lanes that may negatively impact the FV's safety (Dou et al, 2016).

Time-to-collision (TTC) calculates the urgency of lane change. It is the time it would take for vehicles to collide if the following vehicle does not make an adjustment maneuver, i.e., TTC equals the longitudinal range ( $X$-Range in NDS data) between the LCV and the FV divided by their relative speed. Results show that more than $70 \%$ of lane change events' minimum TTC occurred between LCV initiation and the cross-lane point (yellow phase in Fig. 8). That is, in most braking-required events, the FV driver maintains a safe distance by braking before the LCV inserts into its lane. This evidence of human drivers' ability to predict danger and adopt safe, suitable, and timely strategies suggests that ADAS and CAVs can learn a similar response (Casner et al, 2016). For example, a Level 3 (Society of Automotive Engineers (SAE) levels) autonomous vehicle should be able to make appropriate decisions and maintain steady control of the vehicle when it recognizes the surrounding vehicles' intentions.

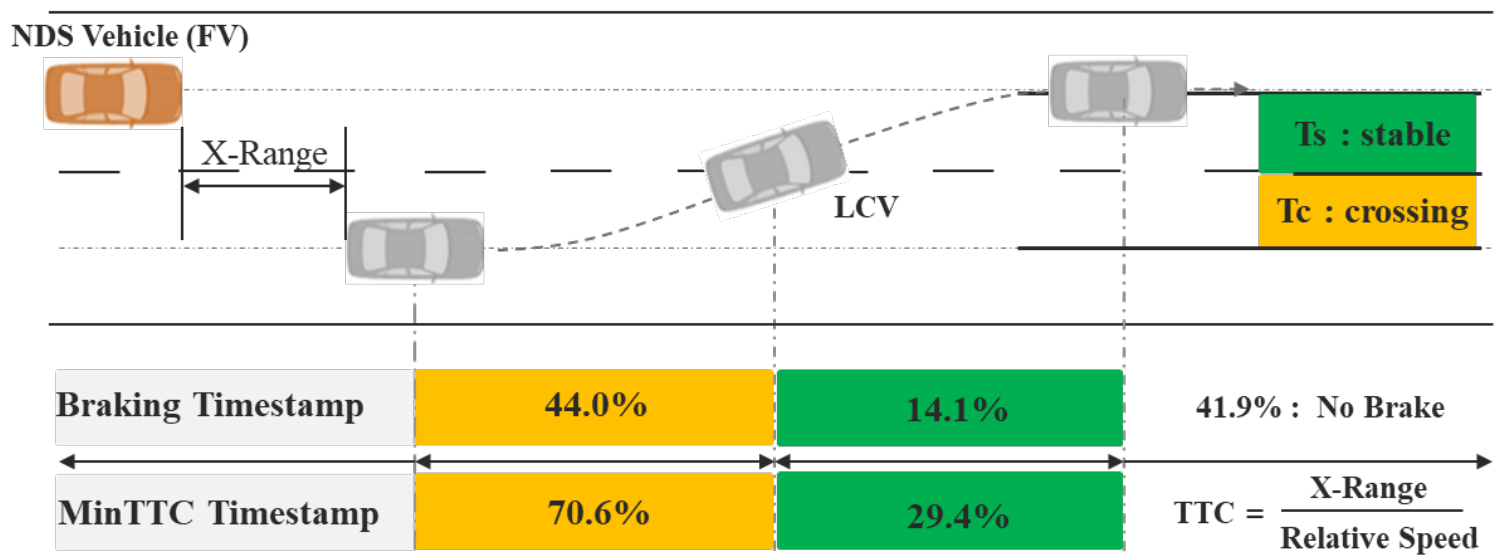

Fig. 8 Braking, minimum TTC and urgency of lane changes. Note: Yellow $=$ initiation to cross-lane; green $=$ cross-lane to stabilization .

Following Olsen et al. (2002), this study calculated TTC for all braking events and classified lane change urgency on a 4-point rating scale (measured by TTC, in second). As shown in Table 4, most FV drivers brake at an urgency of 1, consistent with the observation that FV drivers tend to be cautious when perceiving the CV's intention, while only $5.3 \%$ of drivers waited until TTC was less than $3 \mathrm{~s}$. According to the statistical result, the 95th percentile is $2.8 \mathrm{~s}$ and the 90th percentile is $4.7 \mathrm{~s}$, TTC $<4.7 \mathrm{~s}$ therefore may be an appropriate reference value for ADAS. A warning to brake with a threshold set below $4.7 \mathrm{~s}$ is potentially dangerous, 
as some drivers cannot respond in time, whereas many drivers will ignore a warning set more than $4.7 \mathrm{~s}$ (e.g., $6 \mathrm{~s}$ ). With a warning set at $4.7 \mathrm{~s}$, a braking maneuver could be executed more easily and safely. Our results are similar to the findings of Chen et al. (2015). Their study showed that the frequency and TTC in lane change events varied by different urgency. Meanwhile, Chen et al. (2015) also emphasized that the warning threshold for FCW systems needs to adapt to current vehicle speed and various urgency.

Table 4

9 Braking at different urgency of cut-ins.

\begin{tabular}{cccc}
\hline Urgency Scale & TTC Range & Severity & Braking Percentage \\
\hline 1 & TTC $>5.5 \mathrm{~s}$ & Non-urgent & $83.5 \%$ \\
\hline 2 & $3 \mathrm{~s}<\mathrm{TTC}<5.5 \mathrm{~s}$ & Urgent & $11.2 \%$ \\
\hline 3 & $1 \mathrm{~s}<\mathrm{TTC}<3 \mathrm{~s}$ & Forced & $5.2 \%$ \\
\hline 4 & $\mathrm{TTC}<1 \mathrm{~s}$ & Critical $/$ Near Crash & $0.1 \%$ \\
\hline
\end{tabular}

10

\section{Summary and conclusion}

This study is one of the first to examine drivers' lane change behavior comprehensively, that is, with a focus on the three core components of gap acceptance, duration and impact on the following vehicle. An innovative automatic extraction algorithm was developed to obtain lane change events from the Shanghai Naturalistic Driving Study database. All 5,339 events were used to identify the variables influencing lane change decision-making, execution, and interactions with other vehicles in combination, in an endeavor to provide a new perspective on the entirety of the lane change process.

The first important component of lane change behavior is gap acceptance, which is crucial to understanding a driver's decision to change lanes. As descriptive statistics confirmed that driver decision is influenced by motivation (Toledo et al., 2003; Choudhury and Farheen, 2005) and road type, two three-level mixed-effects linear models were developed to analyze lead and lag gap. The developed models demonstrated that traffic density, weather, light conditions, vehicle type, relative speed and acceleration all had significant effects on lane change decisionmaking.

Duration is another crucial component of the execution phase of lane change, and the current practice of omitting of lane-change duration from microsimulation tools may have a significant effect on simulation outputs. Simulations generally consider duration as nearinstantaneous or assign it a constant value, yet the duration of lane change has considerable range. As the wide range is due to the variety of possible lane-change scenarios, identifying the variables is essential. However, unlike lead and lag gap, duration is not significantly influenced by motivations, but is significantly influenced by traffic density, light conditions, lag gap, relative speed, and acceleration of the following vehicle as well as the lane-changing vehicle.

To better understand lane change impact on the following vehicle, speed change rate, braking timestamp, and time-to-collision (TTC) were used to measure the FV response. Although results demonstrated that the majority of lane changes have limited impact, the percentages of abrupt deceleration and acceleration are not insignificant, and can affect the larger traffic flow as well as the FV. Additionally, the observation that $20 \%$ of Chinese drivers appear to accelerate specifically to prevent cut-in suggests an aggressive driving style, noted in other studies (Wang et al, 2016), that is cause for concern. 
Applications of this study, of course, extend beyond China. Influencing variables for other populations might be compared with this study's results, for example, but a primary application is in providing references for microsimulation software. Results from our models with real road data suggest that simulated gap acceptance and duration thresholds should be set according to a variety of conditions. For example, road type and motivation parameters can be used to simulate various lane change trajectories in different scenarios. This study's results are also useful in the ongoing development of ADAS and CAVs. The fact that $44.0 \%$ of FV drivers brake when the LCV initiates its movement indicates that ADAS must consider movement of vehicles in adjacent lanes as well as the driver's own lane. Additionally, CAV controlling can incorporate findings from this study to become more human-like and comfortable. Since in over $70 \%$ of lane change events, the minimum time-to-collision is shown to occur between initiation and the cross-lane point, CAVs can be designed to brake during that safer phase. Further, the advent of CAV testing in China makes it necessary to consider Chinese lane changing behavior.

Since the lane changing process is complex, any future study should expand to address other variables, such as roadway geometry (i.e., horizontal curvature), the influence of driver psychology on driving styles, and vehicle performance capabilities. Additionally, this study has some limitations that include a passive relatively approach was taken to analyze lane change process, which may not fully capture driver behavior mechanism to some extent. Nevertheless, this paper extends the exploration and development of lane change theory, methodology, and application, and can provide a valuable reference for further research.

\section{Acknowledgements}

This study was jointly sponsored by the Chinese National Science Foundation (51522810; 51878498), the Science and Technology Commission of Shanghai Municipality (18DZ1200200), and the 111 Project (B17032). The authors are grateful to Barbara Rau Kyle for her helpful edit.

\section{References}

Ahmed, K. I., Ben-Akiva, M., Koutsopoulos, H. N., Mishalani, R. G., 1996. Models of freeway lane changing and gap acceptance behavior. Proceedings of the 13rd International Symposium on the Theory of Traffic Flow and Transportation, 24-26.

Al-Kaisy, A. F., Hall, F. L., Reisman, E. S., 2002. Developing passenger car equivalents for heavy vehicles on freeways during queue discharge flow. Transportation Research Part A: Policy and Practice, 36 (8), 725-742.

Al-Kaisy, A. F., Jung, Y., 2004. Examining the effect of heavy vehicles on traffic flow during congestion. Road and Transport Research, 13(4), 3-14.

Annual Statistic Yearbook of Road Traffic Accidents in China (2015), 2016. Traffic Management Bureau of the Public Security Ministry, the People's Republic of China.

APA Balal, E., Cheu, R. L., Sarkodie-Gyan, T., 2016. A binary decision model for discretionary lane changing move based on fuzzy inference system. Transportation Research Part C: Emerging Technologies 67, 47-61.

Bascunana, J. L., 1995. Analysis of lane change crash avoidance. SAE Technical Paper.

Bham, G. H., 2009. Estimating driver mandatory lane change behavior on a multilane freeway. Presented at 88th Annual Meeting of the Transportation Research Board, Washington, 
D.C.

Cao, P., Hu, Y., Miwa, T., Wakita, Y., Morikawa, T., Liu, X, 2017. An optimal mandatory lane change decision model for autonomous vehicles in urban arterials. Journal of Intelligent Transportation Systems 21(4), 271-284.

Casner, S. M., Hutchins, E. L., Norman, D., 2016. The challenges of partially automated driving. Communications of the Acm 59(5), 70-71.

Cassidy, M. J., Bertini, R. L., 1999. Some traffic features at freeway bottlenecks. Transportation Research Part B: Methodological 33(1), 25-42.

Chen, R., Kusano, K. D., Gabler, H. C., 2015. Driver behavior during overtaking maneuvers from the 100-car naturalistic driving study. Traffic Injury Prevention, 16(sup2), S176S181.

Chen, Z., Qin, X., Shaon, M. R. R., 2018. Modeling lane-change related crashes with lanespecific real-time traffic and weather data. Journal of Intelligent Transportation Systems 22 (4), 291-300.

Choudhury, Farheen, C., 2005. Modeling lane-changing behavior in presence of exclusive lane. Massachusetts Institute of Technology.

Coifman, B., Krishnamurthy, S., Wang, X., 2005. Lane-change maneuvers consuming freeway capacity. Traffic \& Granular Flow, 3-14.

Cooper, J. M., Vladisavljevic, I., Strayer, D.L., Martin, P.T., 2007. Drivers' lane changing behavior while conversing on a cell phone in a variable density simulated highway environment. Presented at 86th Annual Meeting of the Transportation Research Board, Washington, D.C.

Deligianni, S. P., Quddus, M., Morris, A., 2017. Analyzing and modeling drivers' deceleration behavior from normal driving. Transportation Research Record: Journal of the Transportation Research Board 2663, 134-141.

Dissanayake, S., Lu, J. J., Ping, Y. I., 2002. Driver age differences in day and night gap acceptance capabilities. IATSS Research, 26 (1), 71-79.

Dou, Y., Ni, D., Wang, Z., Wang, J., Yan, F., 2016. Strategic car-following gap model considering the effect of cut-ins from adjacent lanes. IET Intelligent Transport Systems 10(10), 658-665.

Gipps, P. G., 1986. A model for the structure of lane-changing decisions. Transportation Research: Part B 20(5), 403-414.

Gurupackiam, S., Jr, S. L. J., 2012. Empirical study of accepted gap and lane change duration within arterial traffic under recurrent and non-recurrent congestion. International Journal for Traffic \& Transport Engineering 2(4), 306-322.

Hanowski, R. J., 2000. The impact of local/short haul operations on driver fatigue. Virginia Polytechnic Institute and State University, Blacksburg.

Herman, R., Weiss, G., 1961. Comments on the highway-crossing problem. Operations Research 9(6), 828-840.

Hill, C., Elefteriadou, L., 2013. Exploration of lane changing behavior on freeways. Presented at 92nd Annual Meeting of the Transportation Research Board, Washington, D.C.

Hou, Y., Edara, P., Sun, C., 2015. Situation assessment and decision making for lane change assistance using ensemble learning methods. Expert Systems with Applications 42 (8), 3875-3882.

Kayvan, A., Moridpour, S., Young, W., Wang, Y., Sarvi, M., 2011. Investigating heavy vehicle 
lane changing maneuvers. Presented at 90th Annual Meeting of the Transportation Research Board, Washington, D.C.

Lee, J., Park, M., Yeo, H., 2016. A probability model for discretionary lane changes in highways. KSCE Journal of Civil Engineering 20 (7), 1-9.

Lindgren, A., Chen, F. P., Jordan, W., Zhang, H., 2008. Requirements for the design of advanced driver assistance systems - the differences between Swedish and Chinese drivers. International Journal of Design 2(2), 41-54.

Man, L. H., 2007. Studies on lateral control and lane changing algorithms for application in autonomous vehicles. Hong Kong Polytechnic University (People's Republic of China).

Mauch, M., Cassidy, M. J., 2002. Freeway traffic oscillations: observations and predictions. Proceedings of the 15th international symposium on transportation and traffic theory, Adelaide, Australia, 653-674.

Moridpour, S., Sarvi, M., Rose, G., 2010. Modeling the lane-changing execution of multiclass vehicles under heavy traffic conditions. Transportation Research Record: Journal of the Transportation Research Board 2161(1), 11-19.

Nie, J., Zhang, J., Wan, X., Ding, W., Ran, B., 2017. Modelling of vehicle interaction behavior during discretionary lane-changing preparation process on freeway. Presented at 96th Annual Meeting of the Transportation Research Board, Washington, D.C.

Olsen, E. C. B., Lee, S. E., Wierwille, W. W., Goodman M. J., 2002. Analysis of distribution, frequency, and duration of naturalistic lane changes. Human Factors \& Ergonomics Society Annual Meeting Proceedings 46(22), 1789-1793.

Park, M., Jang, K., Lee, J., Yeo, H., 2015. Logistic regression model for discretionary lane changing under congested traffic. Transportmetrica A: Transport Science 11(4), 333-344.

Regan, M. A., Williamson, A., Grzebieta, R., Tao, L., 2012. Naturalistic driving studies: literature review and planning for the Australian naturalistic driving study. Australasian College of Road Safety Conference, Sydney, New South Wales, Australia.

Salvucci, D. D., Liu, A., 2002. The time course of a lane change: driver control and eye movement behavior. Transportation Research Part F: Psychology \& Behavior 5(2), 123 132.

Salvucci, D. D., Mandalia H. M., Kuge N., Yamamura T., 2007. Lane-change detection using a computational driver model. Human Factors: The Journal of the Human Factors and Ergonomics Society 49(3), 532-542.

Singh, K., Li, B., 2012. Discrete choice modelling for traffic densities with lane-change behavior. Procedia - Social and Behavioral Sciences 43, 367-374.

Srivastava, A., Geroliminis, N., 2013. Empirical observations of capacity drop in freeway merges with ramp control and integration in a first-order model. Transportation Research Part C: Emerging Technologies 30, 161-177.

STATA Multilevel Mixed-Effects Reference Manual Release 13. StataCorp LP, College Station, Texas, 2013.

Sultan, B., Brackstone, M., Waterson, B., Boer, E., 2002. Modeling the dynamic cut-ins situation. Transportation Research Record: Journal of the Transportation Research Board 1803(1), 45-51. http://dx.doi.org/10.3141/1803-07.

Toledo, T., Koutsopoulos, H. N., Ben-Akiva, M. E, 2003. Modeling integrated lane-changing behavior. Transportation Research Record: Journal of the Transportation Research Board, 1857(1), 30-38. http://dx.doi.org/10.3141/1857-04. 
1 Toledo, T., Zohar, D., 2007. Modeling duration of lane changes. Transportation Research Record: Journal of the Transportation Research Board 1999(1), 71-78. http://dx.doi.org/10.3141/1857-04.

Wang, M., Duan, L., Wang, J., 2016. Drive advisory system: do Swedish and Chinese drivers appreciate it in the same way? International Journal of Engineering and Technology 8(4), 286-292.

Wang, X., Li, Y., 2016. Characteristics analysis of lane changing behavior based on the naturalistic driving data. Journal of Transport Information \& Safety 34(1), 17-22.

Wang, X., Yang, M., Hurwitz, D., 2019. Analysis of cut-in behavior based on naturalistic driving data. Accident Analysis and Prevention 124, 127-137.

Yang, Q. I., Koutsopoulos, H. N, 1996. A microscopic traffic simulator for evaluation of dynamic traffic management systems. Transportation Research Part C: Emerging Technologies 4(3), 113-129.

Zhang, L., Kovvali, V. G., 2007. Freeway gap acceptance behaviors based on vehicle trajectory analysis. Presented at 86th Annual Meeting of the Transportation Research Board, Washington, D.C.

Zheng, Z., 2014. Recent developments and research needs in modeling lane changing. Transportation Research Part B: Methodological 60(1), 16-32.

Zhou, J., Peng, H., 2015. Range policy of adaptive cruise control vehicles for improved flow stability and string stability. IEEE Transactions on Intelligent Transportation Systems 6(2), 229-237.

Zhu, M., Wang, X., Tarko, A., Fang, S., 2018. Modeling car-following behavior on urban expressways in Shanghai: a naturalistic driving study. Transportation Research Part C: Emerging Technologies 93, 425-445. 